\title{
Modeling charge transport in gold nanogranular films
}

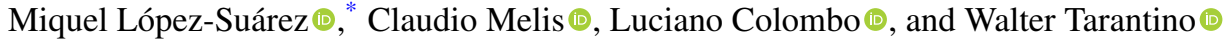 \\ Dipartimento di Fisica, Università degli Studi di Cagliari, Cittadella Universitaria, I-09042 Monserrato, Cagliari, Italy
}

(Received 26 July 2021; accepted 16 November 2021; published 10 December 2021)

\begin{abstract}
Cluster-assembled metallic films show interesting electrical properties, both in the near-percolation regime, when deposited clusters do not form a complete layer yet, and when the film thickness is well above the electrical percolation threshold. Correctly estimating their electrical conductivity is crucial, but, particularly for the latter regime, standard theoretical tools are not quite adequate. We therefore developed a procedure based on an atomically informed mesoscopic model in which ab initio estimates of electronic transport at the nanoscale are used to reconstruct the conductivity of nanogranular gold films generated by molecular dynamics. An equivalent resistor network is developed, appropriately accounting for ballistic transport. The method is shown to correctly capture the nonmonotonic behavior of the conductivity as a function of the film thickness, namely, a signature feature of nanogranular films.
\end{abstract}

DOI: 10.1103/PhysRevMaterials.5.126001

\section{INTRODUCTION}

Cluster-assembled metallic (or, simply, "nanogranular") films may play an important role in the development of emerging technologies. In particular, they show a resistive switching behavior [1] that can be exploited in the fabrication of electrical devices able to process and store data in the same physical unit [2-4], as requested by the neuromorphic computing paradigm $[5,6]$. Such behavior emerges in the nearpercolation regime [7-9], when deposited clusters do not form a complete layer of the film yet, as well as when the film thickness is well above the electrical percolation threshold [10-13]. In particular, for this latter situation a well-established explanation of the underlying physical mechanisms is still missing.

Atomistic simulations may help to get insights on the microscopic mechanisms responsible for such phenomena. Correctly estimating the electrical conductivity becomes therefore crucial $[13,14]$. To this end, we developed an atomically informed mesoscopic model which provides accurate conductivity estimates for systems composed by interconnected gold nanoclusters.

The conductivity of nanogranular films is strongly affected by the high degree of porosity and defects in the metallic component. As the first step, we resort to atomistic simulations based on molecular dynamics to create realistic structures that capture the complexity of nanogranular films at the nanoscopic scale, a method that has been successfully used in the past to analyze the morphology and mechanical properties of such systems [15]. Provided with a realistic representation

\footnotetext{
*mlopez@dsf.unica.it
}

Published by the American Physical Society under the terms of the Creative Commons Attribution 4.0 International license. Further distribution of this work must maintain attribution to the author(s) and the published article's title, journal citation, and DOI. of the atomic-scale complexity of the system, we proceed by calculating its conductance by means of an equivalent resistor network (ERN). Within such an approach, a system is typically approximated with a network of interconnected resistors whose impedance is determined by its local values; the overall resistance is therefore calculated using Kirchhoff's circuit laws applied to the network.

The typical scale of the inhomogeneities of a nanogranular film is, however, comparable to that of the electron mean free path, $l_{e}$, in the corresponding crystalline phase, i.e., $l_{e}=$ $37.5 \mathrm{~nm}$ for $\mathrm{Au}$ [16]. Specializing in films with thickness well beyond the percolation threshold, we include in the ERN the ballistic component of electronic transport, which dominates at the length scale at which inhomogeneities occur, according to the following picture. Inhomogeneities in the metallic component of a nanogranular film are mainly due to the cluster landing impacts occurring during the deposition stage. They can be characterized as layers of highly disordered (amorphous) matter either between adjacent clusters or within the clusters themselves. We therefore model the metallic component as a collection of amorphous regions mixed with pristine crystalline ones and assume that electronic transport within each region and between regions of the same phase (whose length scale is typically just of a few nanometers) is mainly ballistic, while between regions of different phase the transport is diffusive. Such a picture is encoded in an ERN by requiring that resistors contribute to the overall resistance either ballistically or diffusively, whether they connect regions with the same or different phases, respectively. While the diffusivelike behavior is readily obtained by letting the resistors abide by the classical Kirchhoff circuit laws, the ballisticlike behavior is enforced by assigning to each resistor a value of resistance that does not simply depend on the local structure of the system but on the entire region it belongs to and reflects the size scaling typical of ballistic transport.

Accurate characterizations of the ballistic component of electronic transport in metal nanostructures can be obtained 


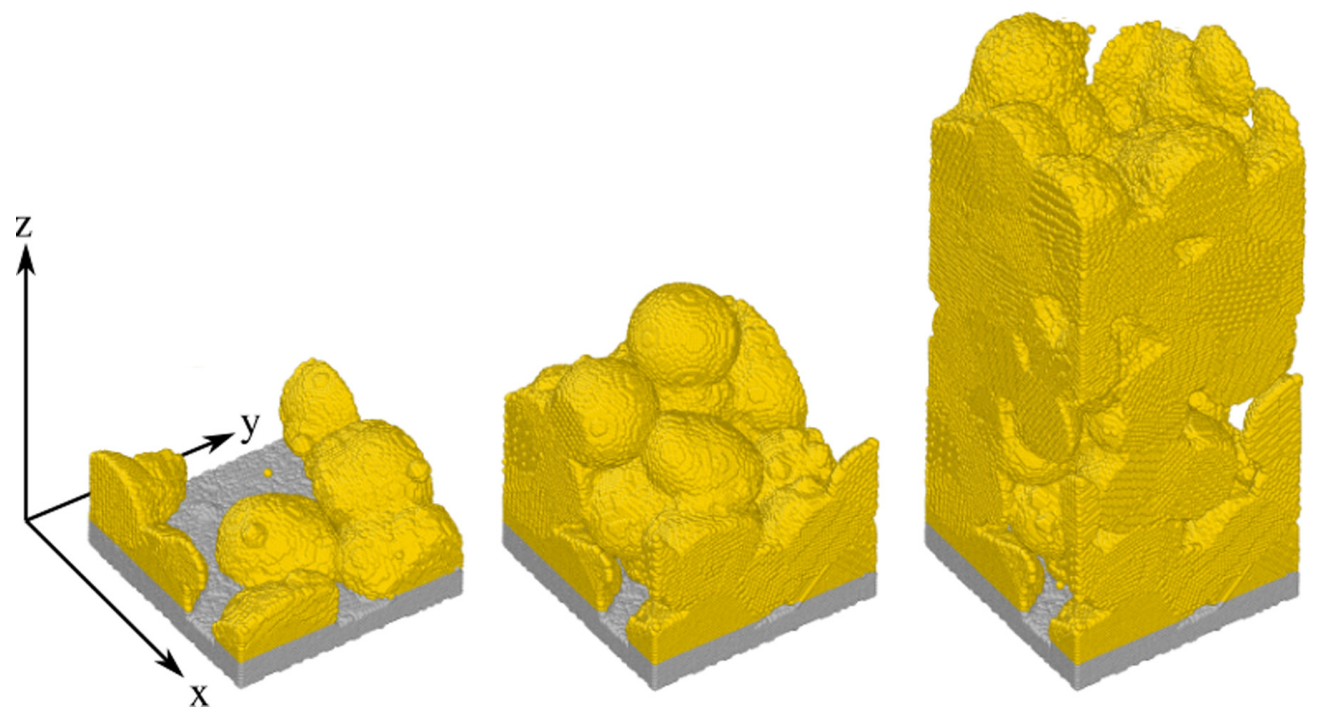

FIG. 1. View of the film growth at three different deposition stages. Left: Just a few Au clusters landed on the substrate (grey atoms). The corresponding thickness of the sample is $<6 \mathrm{~nm}$ and no percolation path connecting two opposite sides of the sample has been created yet. Middle: The creation of the first percolation paths is already achieved, corresponding to a film thickness of $20 \mathrm{~nm}$. Right: The film in an advanced growth stage with a film thickness $>60 \mathrm{~nm}$.

using $a b$ initio methods [17]. In particular, we use density functional theory (DFT) combined with nonequilibrium Green's function (NEGF) techniques (i) to study ballistic transport in structures mimicking the inhomogeneities found in the nanogranular film and (ii) to determine appropriate values of resistance for the ERN. Provided with such an input, the ERN can be finally used to get an estimate of the conductance of the entire simulated system.

To demonstrate the robustness of our procedure, we have simulated the growth of a nanosized sample of a nanogranular film assembled by cluster deposition, close to the experimental conditions of Ref. [10], and calculated its resistance at various stages of growth.

The paper is organized as follows: In Sec. II we describe the methodology used to simulate the growth of the nanogranular Au film by means of classical molecular dynamics (MD). In Sec. III we discuss the procedure to accurately estimate the conductance of the specific gold microstructures observed in the simulated film. In Sec. IV the ballistic ERN used to compute the total resistance of the film is presented. Finally, in Sec. $\mathrm{V}$ we present the results provided by our electrical model and compare them to a set of experimental results.

\section{SIMULATED FILM GROWTH AND STRUCTURAL ANALYSIS}

The cluster-assembled metallic film was obtained by simulating the multiple landing of 210 Au clusters deposited in six different steps [15], by classical MD. All the gold clusters were first thermalized at $300 \mathrm{~K}$ for $150 \mathrm{ps}$. The size population of the clusters was constructed with $70 \%$ of the clusters of diameter $8.8 \mathrm{~nm}$ and $30 \%$ of diameter $1.3 \mathrm{~nm}$, thus reproducing the experimental size distribution [13].

As for the growth process, the first $35 \mathrm{Au}$ clusters were deposited at random positions and normal impact direction on top of the substrate. The average kinetic energies per atom of the landing clusters were fixed to $0.25 \mathrm{eV} /$ atom consistently with the results obtained from the experiments [9]. Periodic boundary conditions were applied in the in-plane directions normal to the growth one. Finally, the clusters were left free to evolve according to Newtonian dynamics. The snapshots of the film corresponding to three different deposition stages are displayed in Fig. 1: after the first deposition steps we observe a film characterized by isolated grains, with most of the substrate surface being unoccupied. We can observe in the subsequent deposition steps the formation of cavities giving rise to the expected film porosity, i.e., $\sim 30 \%$.

MD simulations have been performed using the LAMMPS code [18], integrating the equations of motion by the velocity Verlet algorithm. The Nosé-Hoover thermostat with relaxation time equal to $100 \mathrm{fs}$ was used to control the temperature. The Au-Au interactions were sampled using a 12-6 LennardJones potential with a cutoff at $0.8 \mathrm{~nm}$. The Lennard-Jones parameters have been optimized in order to reproduce several properties such as surface tension density in good agreement with experiment [19], i.e., $\epsilon=5.29 \mathrm{eV}$ and $\sigma=2.62904 \AA$. The Au substrate (grey atoms in Fig. 1) with dimensions of $24.5 \times 24.5 \times 5 \mathrm{~nm}^{3}$ was constructed with the (111) surface exposed to the deposition of the clusters. The four bottom layers were kept fixed in order to mimic a bulk material and a slab region ( $1.7 \mathrm{~nm}$ thick) adjacent to the fixed slab was thermalized at room temperature.

Figure 2 displays a gold grain landed on the substrate; while before landing, by construction, the cluster is perfectly spherical, the collision with the substrate strongly affects its shape and structure. We observe in the deposited film two kinds of atomic arrangements: cubic (fcc) and noncubic (non-fcc) gold. A polyhedral template matching (PTM) analysis performed with OVITO [20], a scientific analysis software for molecular simulation models, allows to distinguish between those $\mathrm{Au}$ atoms sitting in fcc sites and those which are not (related to planar and bulk defects). In the left panel 


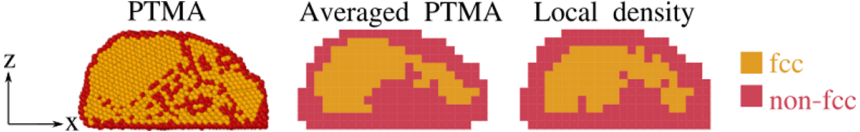

FIG. 2. An $x z$ view of a single Au deposited cluster with radius $r=4.4 \mathrm{~nm}$ after landing. The collision against the substrate strongly modifies its original spherical shape, creating different microstructural defects. Left: A polyhedral template matching analysis allows to localize with atomic resolution the distribution of the defects in the cluster. Orange atoms are found in fcc sites while red ones cannot be classified as such. Middle: The PTM analysis is averaged over the grid elements of the ERN allowing to distinguish fcc (orange) and non-fcc (red) regions. Right: Distribution of fcc (orange) and non-fcc regions (red) based on the local density of the granule showing a good agreement with the averaged PTM analysis.

of Fig. 2 we show a section of the PTM analysis performed on the gold cluster after landing. For the sake of clarity we have excluded from the analysis those atoms belonging to the substrate. We observe that, due to the collision with the substrate, the fcc symmetry is broken, thus originating regions with different crystal structure. These defects are local and separate different fcc regions (orange colored atoms) within a single grain. In addition to that, the fcc symmetry is also broken at the surface of the cluster, creating a non-fcc shell all around the grain. The shell is found before and after the collision; thus, it is not produced by the exceeding kinetic energy after landing. However, we do observe an increase of the shell thickness after cluster landing. Moreover, the nonfcc shell is responsible for having non-fcc layers separating adjacent deposited clusters in the film.

The PTM analysis provides very accurate atomically resolved structural phase maps of the clusters assembled to form the film. However, its heavy computational cost prevents from using PTM to distinguish the different phases during the evolution of the simulated film, which is made up of more than $10^{6}$ atoms. Therefore, we rather measure the local atomic density, $n_{l}$, of the film: we observe that the presence of defects induces a slight increase in the $\mathrm{Au}-\mathrm{Au}$ bond length which shifts from $b_{m}=2.89 \AA$ for fcc Au regions to $b_{m}=2.99 \AA$ for non-fcc ones. This effect can be seen in Fig. 3(a), where the radial distribution function calculated on a subregion of the granule containing fcc atoms (orange line) and non-fcc atoms (red line) is shown. A more evident effect is the broadening of the peaks for the non-fcc region. The upward shift of the first peak for non-fcc regions is directly translated to a decrease of the local density that we define as $n_{l}=N_{r} / V_{r}$, where $N_{r}$ is the number of gold atoms contained in that particular region of volume $V_{r}$. Two specific local density threshold values, $n_{v}$ and $n_{c}$, are used to set the density ranges corresponding to vacuum, non-fcc, and fcc gold. We consider a region to be vacuum if its local density falls below $n_{v}$, while cubic gold is defined as $n_{l}>n_{c}$. Finally, noncubic gold corresponds to $n_{v}<n_{l}<n_{c}$. In order to evaluate the agreement between the two methods we have averaged the former PTM analysis over the regions on which the local density approach is performed, i.e., a regular grid with element size $5 \times 5 \times 5 \AA^{3}$; thus $V_{r}=0.125 \mathrm{~nm}^{3}$. By doing so we obtain the color map displayed in the middle panel of Fig. 2. In the right-hand panel,
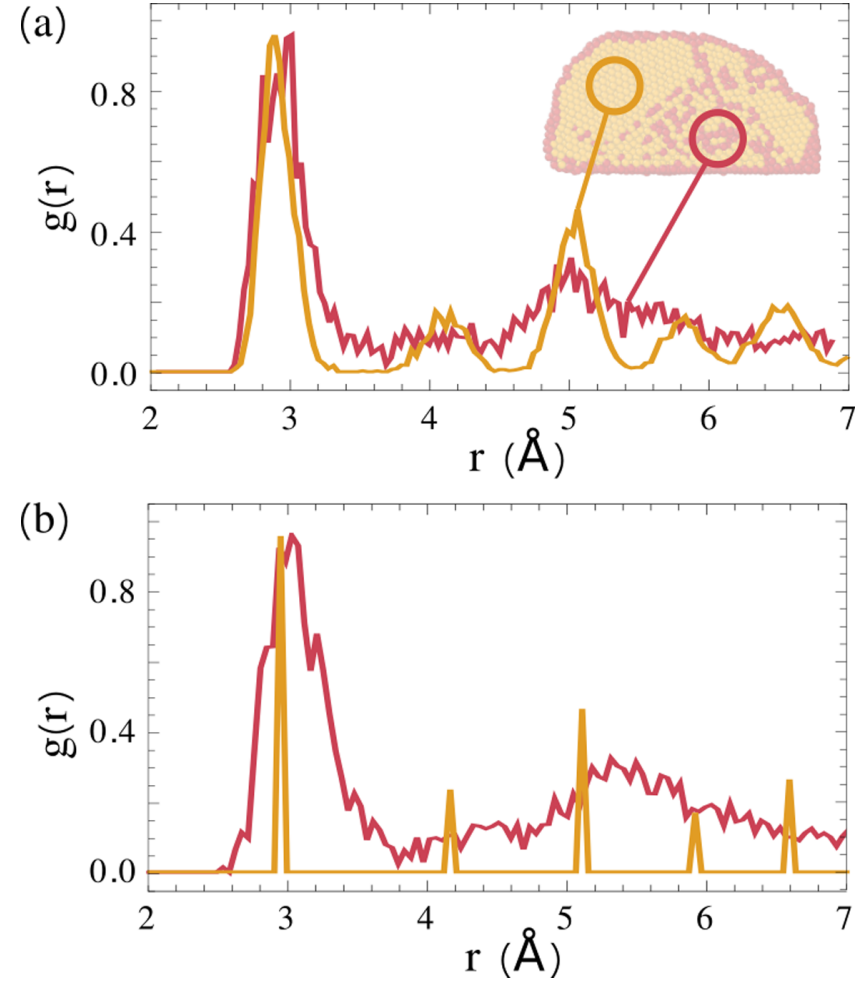

FIG. 3. (a) Pair correlation function $g(r)$ computed on the highlighted regions of the cluster showing a first peak at $r=2.89 \AA$ and $r=2.99 \AA$ for fcc (orange) and non-fcc (red) phase, respectively. (b) $g(r)$ for the DFT simulation cells showing a main peak placed at $r=2.95 \AA$ and $r=3.04 \AA$ for fcc and non-fcc, respectively. The cells are prepared to match the ratio obtained in the $g(r)$ distributions from the simulated sample shown in (a), i.e., $b_{\text {non-fcc }} / b_{\text {fcc }}=1.03$.

the corresponding local density map is shown with $n_{v}=$ 0.0005 atoms $/ \AA^{3}$ and $n_{c}=0.048$ atoms $/ \AA^{3}$. Despite the much lower computational cost a good agreement in the ratios between the fcc, non-fcc, and vacuum occupied volume and the total volume is observed. A side effect is a slight overestimation of the non-fcc shell's thickness for the local density approach.

\section{ELECTRON TRANSPORT IN GOLD NANOJUNCTIONS}

The ballistic component is supposed to dominate the electronic transport within individual nanoparticles at the considered length scales. Other transport mechanisms such as tunneling and hopping are not included since a strong-coupling regime [21] is expected at the considered temperature and lengths, here neither Coulomb interaction nor quantum interference effects occur (see the Supplemental Material [22]). We assume the two individuated gold phases, i.e., fcc and non-fcc, to have different electronic transport characteristics. This is justified by the fact that non-fcc Au regions are characterized by a lack of symmetry that effectively reduces the number of opened conduction channels for ballistic transport [23]. Moreover, we also assume that the estimation of the ballistic conductance of the two phases is sufficiently accurate to build up a reliable resistive model. The conductance of fcc and non-fcc Au is estimated by a blended NEGF-DFT 
(a)

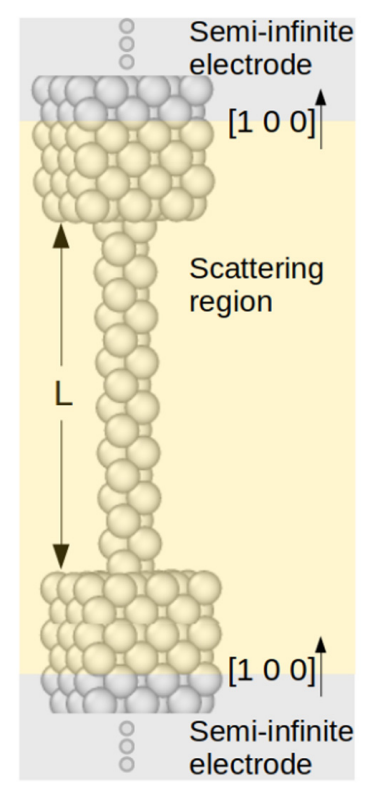

(b)

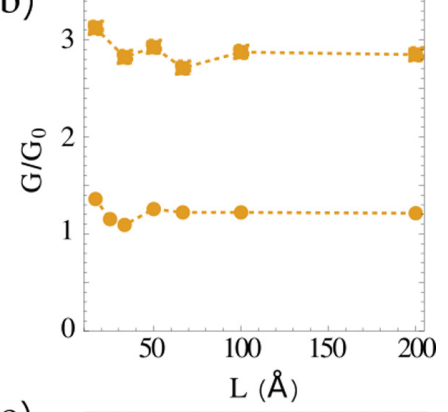

(c)

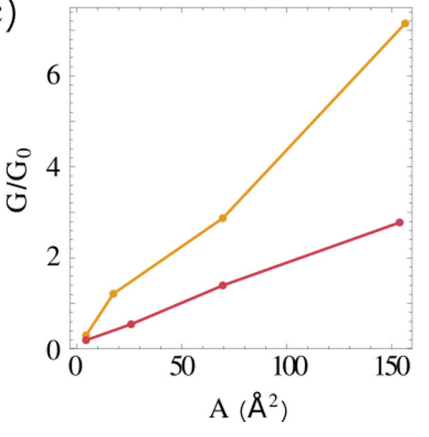

FIG. 4. (a) Schematic view of the simulated device. Two semiinfinite [100] Au electrodes (grey atoms) and the scattering region formed by a repetition of the electrodes plus the gold junction (yellow atoms). (b) Computed conductance $G$ for fcc-Au junctions with different lengths, $L$, for two different wire cross sections, $A$ : plateaus at $1.2 G_{0}$ and $2.9 G_{0}$ are obtained in agreement with Ref. [26]. (c) Computed conductance, $G / G_{0}$, for different cross section $A$ for fcc (orange line) and non-fcc (red line) Au junctions with $L=100 \AA$.

approach. In particular, the computation of the conductance of gold nanosized systems [17,24,25] has been boosted by recent experiments on electronic properties of atomic-sized gold structures [26-32]. The remarkable agreement between estimates and experimental values for different lengths, cross sections, and crystallographic orientations proves the accuracy of this approach in the study of electron transport in gold systems at the nanoscale.

Using such an approach, we are therefore able to perform a comprehensive study of the conductivity of a two-terminal device containing gold junctions mimicking the structures individuated in the structural analysis of the MD samples, i.e., fcc and non-fcc. More specifically, in order to compute the conductance of gold junctions that might be representative of those found in the simulated film, we proceed as follows: We first set the crystallographic orientation of the device electrodes of the two-terminal device, once for all. Atomic-scale Au junctions are built up with different lengths, $L$, and cross sections, $A$, in between the two electrodes. To mimic the fcc phase, we require the atoms belonging to the central scattering region to keep the crystallographic orientation of the electrodes [as shown in Fig. 4(a)], while non-fcc ones are required to (i) present no specific crystallographic orientation under the PTM analysis and (ii) to present a broader radial distribution function than fcc as observed in Fig. 3(a). This is equivalent to requiring each added atomic layer to change orientation with respect to the previous one. In such a scheme, the first

allows electrons to see the symmetry of the lattice along the device, while the latter incorporates the nonhomogeneity of the medium found in the deposited clusters. The $g(r)$ for fcc and non-fcc gold junctions are displayed in Fig. 3(b). Another possible approach to evaluate the conductance of the defects individuated in the simulated film is to simply carve out from the film those regions we are interested in. The reason to avoid this approach is the fact that the nonhomogeneities found in the film extend only for few atomic layers, while the required calculations that allow to specify a unique value for the conductance depending on $A$ and $L$ require the consideration of lengths and cross sections beyond that limit [see length scales in Figs. 4(b) and 4(c)].

We describe the gold electronic structure self-consistently using DFT within the generalized gradient approximation (GGA) as implemented in the SIESTA package [33]. Core electrons are modeled with Troullier-Martins nonlocal pseudopotentials, while the valence electrons are expanded with a double $-\zeta$ basis set. The mesh cutoff is $300 \mathrm{Ry}$ and a $10 \times 10 \times 10 k$-point mesh is used for the four-atom unit cell. We relax all the atomic coordinates till atomic forces are below 0.04 and $0.10 \mathrm{eV} / \AA$ for fcc and non-fcc $\mathrm{Au}$, respectively.

For the conductance calculations, we have used TRANSIESTA [34], which is based on the combination of DFT with the NEGF technique. Therefore, calculations on transport properties are based on the Landauer scheme of elastic scattering probability [35]. Within such a scheme, given a certain bias, $V$, it is possible to compute the current, $I$, after self-consistently solving the NEGF and the electrostatic potential to get the electronic density matrix. The conductance of the device is then computed as $G=I / V$. Semi-infinite $8 \times 8$ 100-Au electrodes, four layers thick, sampled with a converged $k$-point grid of $3 \times 3 \times 20$, are considered.

In the ballistic transport regime the conductance of a material is well known to be independent of the device length. The first step is to compute the conductance, $G$, for fcc gold junctions for different cross sections and increasing lengths ranging from tens to hundreds of angstroms. The computed values of $G$ against the wire length, $L$, are displayed in Fig. 4(b) in units of the quantum of conductance, $G_{0}=$ $0.0000775 \Omega^{-1}$. We observe fluctuations in the computed $G$ values for short wires $(L<50 \AA$ ) while $G$ converges to a constant value for longer wires, as expected for ballistic transport. Many experiments [28,36] and computational works [24,25] have reported an increase of $G$ for wires with increasing $A$, due to the increase in the number of opened conduction channels. A linear relation between $G$ and $A$ is expected with a slope depending on the crystallographic orientation [26]. We obtain values close to $1.2 G_{0}$ and $2.9 G_{0}$ for the two types of gold junctions considered in Fig. 4(b). In particular, as the cross section of a wire oriented along the [100] direction is increased from $1.7 \AA$ (corresponding to 4 unit cells) to $7.0 \AA$ (corresponding to 16 unit cells), an increase of $\Delta G=1.7 G_{0}$ is observed corresponding to the transition from $1.2 G_{0}$ to $2.9 G_{0}$, close to the expected value provided by the simplified free electron model used in Ref. [26], i.e., $1.8 G_{0}$. Figure 4(c) shows the conductance for long wires $(L=100 \AA)$ for increasing cross sections for fcc (orange) and non-fcc Au wires (red). The linear trend for fcc junctions is characterized by a slope of 
(a)

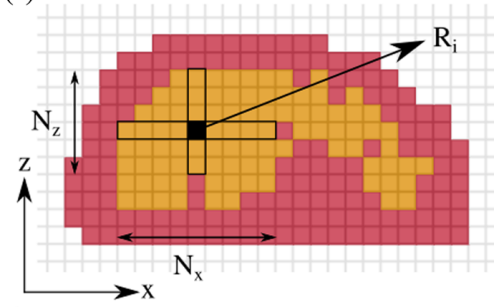

(b)

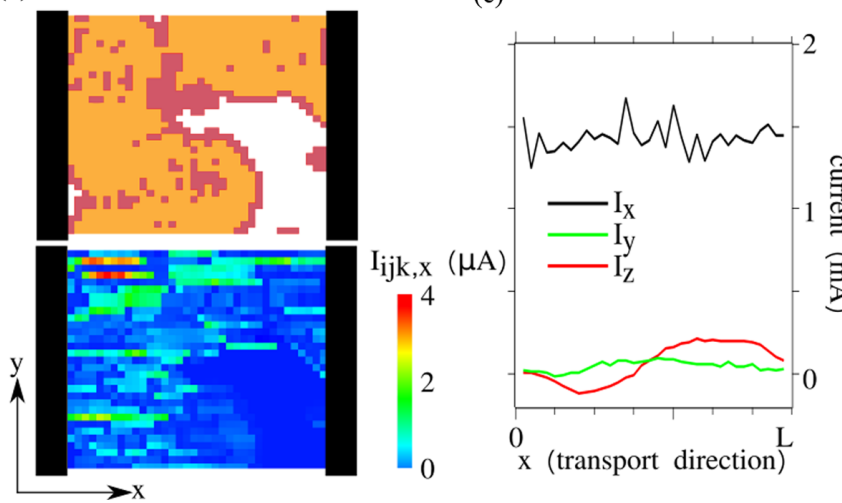

FIG. 5. (a) Left: $x z$ view of the regular 3D grid applied to a single gold cluster. For a given a mesh element, $N_{i}$ is the number of adjacent cells with equal gold phase (fcc or non-fcc) along the $i$ direction. Right: Total resistance along a given transport direction for different combinations of fcc and non-fcc cells. $R_{c}$ and $R_{a}$ stand for the resistance value of a single fcc and non-fcc element, respectively. (b) An $x y$ view of fcc and non-fcc cells of the film at $z=15 \mathrm{~nm}$ and the corresponding $I_{i j k, x}$ map $(k=30)$ obtained from the converged solution of the ERN. Black strips represent the electrodes. (c) The three components of the current vector $\left(I_{x}, I_{y}, I_{z}\right)$ computed along the transport direction.

$0.04 G_{0} / \AA^{2}$ while it is less pronounced for non-fcc junctions, i.e., $0.02 G_{0} / \AA^{2}$. As expected, non-fcc Au junctions are less conductive than fcc ones, for all considered $A$.

\section{EQUIVALENT RESISTOR NETWORK WITH BALLISTIC TRANSPORT}

An ERN model is used to evaluate the electrical conductivity of the simulated film. A three-dimensional (3D) regular grid is superimposed on the deposited film and each ERN cell is filled with one of the following: fcc $\mathrm{Au}$, non-fcc $\mathrm{Au}$, or vacuum depending on the local value of $n_{l}$ as explained in Sec. II. An $x y$ projection of the grid over a single landed gold cluster is shown in Fig. 5(a). We assign to each ERN grid element a resistance value as follows: for vacuum elements this is set to $10^{15} \Omega$, while for Au elements, this is computed by sticking to the geometrical dependencies found in Figs. 4(b) and 4(c). The rationale behind the value chosen for vacuum is to ensure those elements do not contribute to the final resistance value. This can be achieved by setting this value to infinity, which carries numerical issues. Instead, we choose to set this to a sufficiently high value with respect to the fcc and non-fcc ones, both falling in the $\mathrm{k} \Omega$ range.

From the simulated sample we can roughly distinguish two types of interface: interfaces separating two grains with different crystallographic orientation ("fcc-fcc") and those separating ordered grains from disordered regions ("fcc-nonfcc"). In our modeling, the latter type is always recognized as an interface that disrupts the electronic transport so the total resistance of a slab of fcc gold in contact with a nonfcc region is equal to the sum of their resistances, capturing the decoherence of electrons when reaching the interface and the interruption of the ballistic transport. In other words, the interface limits the regions where the electronic transport is considered ballistic. Instead, fcc-fcc interfaces, on the other hand, affect the transport only if the change of symmetry in going from one grain to the other is high enough so the density analysis detects the intermediation of a non-fcc region in between; otherwise the interface is effectively neglected. It must be remarked that clean fcc-fcc interfaces rarely occur, as one can see in Fig. 2.

Thus, for a given mesh element containing $\mathrm{Au}$ atoms in a fcc (non-fcc) phase, we compute the number, $\left(N_{x}, N_{y}, N_{z}\right)$, of consecutive cells along each Cartesian direction containing cubic (noncubic) gold. We then give a unique resistance value for each transport direction as $R_{i}=R_{b} / N_{i}$, where $R_{b}=$ $[G(A)]^{-1}$ and $i=x, y, z$. Doing so we ensure the total resistance of a given chunk of gold does not depend on its length, and only the cross section $A$ determines its final value as expected for ballistic transport. Therefore, given a transport direction, if the total resistance value for a single fcc (non-fcc) mesh element is $R_{c}\left(R_{a}\right)$, the total resistance for $N$ consecutive fcc (non-fcc) cells along that transport direction equals $R_{c}\left(R_{a}\right)$. Instead, the alternative stacking of fcc and non-fcc elements results in a total resistance that equals the sum of the constitutive parts, as represented in Fig. 5(a).

The ERN grid is made up of $\mathrm{I} \times \mathbf{J} \times \mathrm{K}$ elements, the dimensions of which have been chosen so as (i) to minimize the computational cost of solving iteratively the ERN for the considered structures and (ii) to have enough spatial resolution to well resolve the intra- and intergranule structure. We set the element size to $5 \AA$, so the element volume and the minimum resolved area are $0.125 \mathrm{~nm}^{3}$ and $0.25 \mathrm{~nm}^{2}$, respectively. With this the ERN has $50 \times 50 \times \mathrm{K}$ elements with $\mathrm{K}$ increasing at each deposition step in order to include all deposited clusters. Once all the mesh elements count with a resistance value, a finite bias, $V_{\text {bias }}$, across the sample is applied: the voltage is set to $V=V_{\text {bias }}$ for those grid elements belonging to one of the electrodes, while it is set to $V=0 \mathrm{~V}$ otherwise. We have used $V_{\text {bias }}=0.06 \mathrm{~V}$ to generate all the data included in Sec. V. We stress at this point that the $R_{T}$ estimation of the total film resistance produced by the present linear model does not depend on this parameter.

The obtained electrical network is analyzed by solving the Kirchhoff equations. We solve them iteratively updating the node voltages $V_{i j k}$ using the formula

$V_{i j k}=\frac{\frac{V_{i-1, j, k}}{R_{(i-1) j k, x}}+\frac{V_{i+1, j, k}}{R_{i j k, x}}+\frac{V_{i, j-1, k}}{R_{i(j-1) k, y}}+\frac{V_{i, j+1, k}}{R_{i j k, y}}+\frac{V_{i, j, k-1}}{R_{i j(k-1), z}}+\frac{V_{i, j, k+1}}{R_{i j k, z}}}{\frac{1}{R_{(i-1) j k, x}}+\frac{1}{R_{i j k, x}}+\frac{1}{R_{i(j-1) k, y}}+\frac{1}{R_{i j k, y}}+\frac{1}{R_{i j k, z}}+\frac{1}{R_{i j(k-1), z}}}$,

where $R_{i j k, x}$ is the resistance of the $(i, j, k)$ grid element in the $x$ direction, etc., and keeping fixed the electrode voltage. 
Iterations are performed until the variation of the sample total resistance between iteration steps is less than $0.01 \Omega$.

From the node voltages and the resistances, one can calculate the current flowing through the simulated sample. Each $(i, j, k)$ grid element is made up of a three-component current vector $\left\{I_{i j k, x}, I_{i j k, y}, I_{i j k, z}\right\}$. In Fig. 5(b) we show the converged current map at $z=15 \mathrm{~nm}(k=30)$ for the simulated sample at a very advanced growth stage along with the corresponding fcc or non-fcc grid at that film height. The total current vector along the bias direction $[x$ direction in Fig. 5(b)] is computed as $\mathbf{I}=\left(\sum_{j k} I_{i j k, x}, \sum_{j k} I_{i j k, y}, \sum_{j k} I_{i j k, z}\right)$. The three components of the total current vector are displayed in Fig. 5(c). We observe that, after reaching the converged solution of the ERN, the $x$ component of the total current vector, $I_{x}$, fluctuates around a constant value $\left(1.5 \mathrm{~mA}\right.$ with $\left.V_{\text {bias }}=0.06 \mathrm{~V}\right)$ all along the transport direction [black line in right-hand panel of Fig. 5(c)]. For $I_{y}$ and $I_{z}$ we observe the generation of internal currents that cancel each other (see $\sim 0$ values close to the extremes of both curves) so no current gets in or out of the system through the directions normal to the transport one. Finally, we compute the total resistance of the film as $R_{T}=V_{\text {bias }} / \bar{I}$, where $\bar{I}$ is the mean value of the total current flowing through the film along the bias direction.

To reach the converged solution for the ERN represents the most expensive part of the present model in terms of CPU time $(600 \mathrm{~s}$ for a $50 \times 50 \times 75 \mathrm{ERN}$ run in a single core).

\section{RESULTS}

The hierarchy of MD, NEGF-DFT, and ERN models allows to compute the evolution of the film electrical resistance as gold clusters are deposited on the substrate. We have reproduced the percolation curves for the simulated sample and the corresponding data are displayed along with the experimental measurements from Ref. [10] in Fig. 6. We considered two different electrostatic bias conditions by setting the electrodes along the $x$ ( $x$ bias) and $y$ directions ( $y$ bias), as depicted in Fig. 6. The determination of the film thickness, $t$, is performed following the definition used to plot the results of the experiments in Ref. [10]: we count the number of cells containing $\mathrm{Au}$ atoms in the ERN grid, $N_{c}$. Given the sample in-plane dimensions we compute $t_{b}=0.125 \mathrm{~nm}^{3} N_{c} /(24.5 \mathrm{~nm})^{2}$ corresponding to the thickness of a bulk film with the same amount of matter. Finally, the porosity of the sample is introduced to obtain the final thickness value, $t=1.35 t_{b}$. We name $t_{p}$ the film thickness at which the first percolation path is created. For $t<t_{p}$, the film is characterized by isolated clusters and an infinite resistance ( $>10^{13} \Omega$ for numerical reasons). Since the current implementation does not take into account electron tunneling and hopping effects, our data start being meaningful after the creation of the first percolation path, which creates a real contact between the electrodes. We observe the first percolation path occurring at $t_{p, x}=5 \mathrm{~nm}$ and $t_{p, y}=10 \mathrm{~nm}$, for the two considered bias conditions. After that, two growth stages are identified from the resistance-thickness curve [37]. At first, few intergrain electrical contacts exist, producing a film characterized by poorly connected aggregates and $R_{T}$ values in the $1-10 \mathrm{k} \Omega$ range. In this stage, known as the geometrical percolation stage, $R_{T}$ abruptly decreases down to hundreds of $\Omega$ due to the increase in the paths becoming

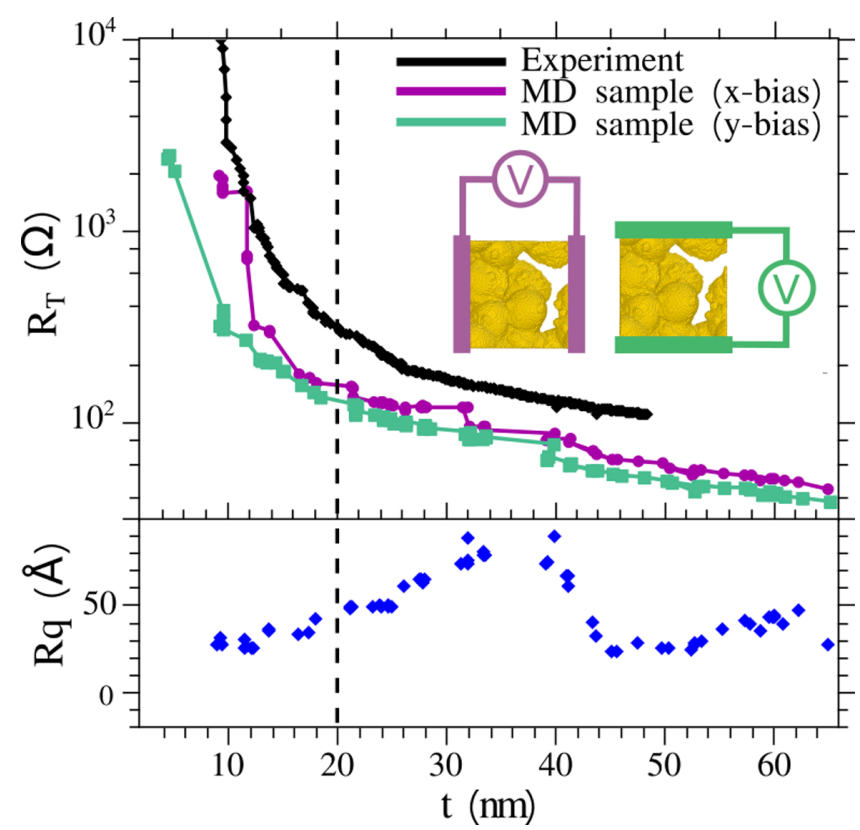

FIG. 6. Top: Percolation curves for the electrical resistance of cluster-assembled films as a function of the film thickness on the $x$ axis (semilogarithmic scale). Bottom: Evolution of the surface film roughness, $R q$, for the simulated sample. The dashed line indicates the $t_{m}$ thickness at which $R_{T} t^{2}$ reaches its minimum.

available for electron transport as clusters land and interconnect. Next, a transition from insulating to ohmic behavior is observed; i.e., $R_{T}$ smoothly decreases as $t$ is increased. This transition is defined as the thickness at which the quantity $R_{T} t^{2}$ reaches its minimum value $t_{m}$ [38,39]. The good agreement achieved for the determination of this parameter is shown in Fig. 7. For all data sets the transition to ohmic behavior is achieved around $t_{m}=20 \mathrm{~nm}$. However, this parameter can suffer huge fluctuations from one sample to another [10]. The predicted $R_{T}$ values show larger fluctuations in comparison to the experimental curve, these being more important at the first percolation steps. The fact that the simulated film

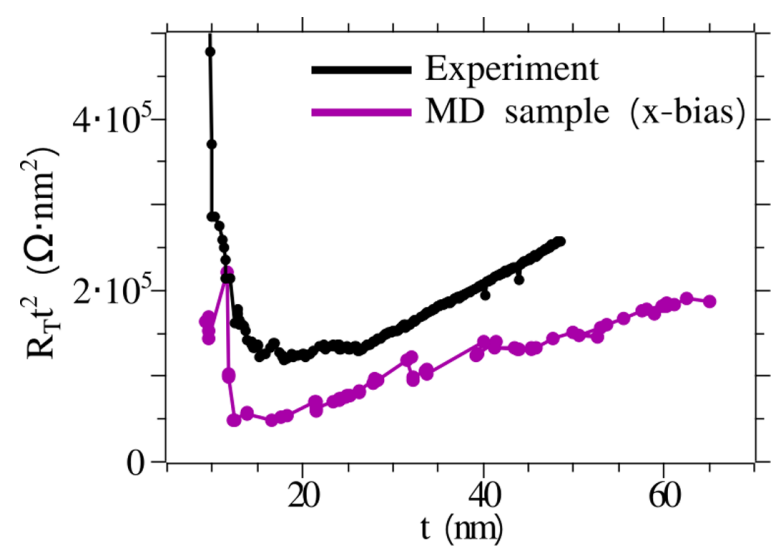

FIG. 7. $R_{T} t^{2}$ as a function of film thickness $t$. The minimum position on the simulated curve is determined with a parabolic fit to be at $20 \mathrm{~nm}$. Black dots correspond to the experimental data and magenta dots to the simulated sample ( $x$ bias). 
has nanoscale dimensions, i.e., $25 \times 25 \mathrm{~nm}^{2}$, make granular effects still visible at all deposition stages and the landing of a single cluster produces huge variations in the computed values. Instead, the experimental curve corresponding to a macroscale sample presents a smoother behavior.

For $t>t_{m}$, the total film resistance further decreases (see Fig. 6). Quantitatively, our electrical model slightly underestimates the total resistance of the film for every deposition step. For instance, the ratio between the computed and the measured value at $t=40 \mathrm{~nm}$ is 0.6 and 0.7 for $x$ bias and $y$ bias, respectively. Both experimental and simulated data follow a power-law decay $\left(R_{T} \propto 1 / t^{\alpha}\right)$ as clusters are deposited and this represents a qualitative agreement with the experimental data set. The experimental curve provides $\alpha_{\text {expt }}=0.9$, implying that the resulting film resistivity, calculated as $\rho=R_{T} t$, should increase instead of remaining constant as expected for bulk materials and also observed for atomically assembled $\mathrm{Au}$ films. The power-law exponents for the two simulated curves are equal to $\alpha_{x}=0.94$ and $\alpha_{y}=0.95$ for that thickness range, fulfilling the condition $\alpha<1$ to observe an increase of $\rho$. Indeed this is a quite remarkable agreement with experimental results.

This effect is often attributed to an increase of the sample roughness as suggested in Refs. [40-42]. In order to support the plausibility of this hypothesis we have computed the roughness of the film surface, $R q$, which we estimate by means of the standard deviation of the film height measured on every $(i, j)$ mesh element as $R q=\sqrt{\frac{\sum^{N}\left(z_{i j}-\bar{z}\right)^{2}}{N-1}}$, where $z_{i j}$ is the height profile of the sample and $\bar{z}$ stands for the mean sample height. Differently from experimental realizations, we do have access to $R q$ at every deposition step so a sound characterization of the film roughness dynamics is achieved. In Fig. 6(b) the evolution of $R q$ is shown, displaying clearly two alternate regimes: one in which clusters are accumulated in few spots of the $(x, y)$ plane producing an increase of $R q$ corresponding to $t<40 \mathrm{~nm}$ and $t>45 \mathrm{~nm}$, and another in which the deposition of few clusters is enough to drastically reduce $R q$, generating void regions within the film which results in the expected film porosity. The former, characterized by a nonuniform deposition of clusters, produces a reduction of the slope of the $R_{T}$ curve, due to the fact that the clusters do not contribute to create new conduction paths. The latter, in which new clusters occupy regions connecting separated gold regions, effectively reduces the film resistance. The periods of these two regimes are expected to depend on the ratio between the sample size and the cluster dimensions, being shorter for smaller ones. The correlation between the modeled film

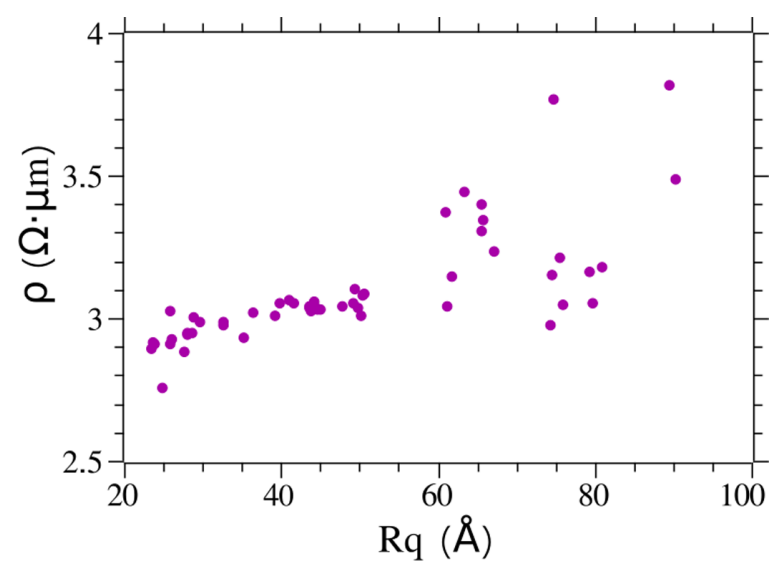

FIG. 8. $\rho$ vs $R q$ curve for the simulated sample for $t>20 \mathrm{~nm}$.

resistivity and its roughness for $t>40 \mathrm{~nm}$ can be observed in Fig. 8. Thus, the present electrical model well captures the role of surface roughness in the evolution of the film growth.

\section{CONCLUSIONS}

We have presented an electrical model able to predict the resistance of cluster-assembled gold films based upon a wellresolved description of their nanostructure and related charge transport at that length scale. By reproducing the deposition of tens of Au clusters on a substrate by means of MD simulations we are able to reproduce the growth of a Au nanogranular film. Next, by using our model at different growth stages we compute the resistance percolation curve and compare the model predictions against experimental values, obtaining a good agreement. In particular, the power-law dependence of the total resistance with the film thickness is well reproduced as well as the nonmonotonic behavior of the film resistivity and its dependence on the film surface roughness. The modeled film is $\sim 30 \%$ more conductive with respect to the experimental one, likely due to the simplified synopsis of transport mechanisms it is based on. Further improvement of the model could be achieved by including tunneling and hopping transport mechanisms to extend the applicability of the model to the $t<t_{p}$ region of the percolation curve.

\section{ACKNOWLEDGMENT}

This work was fully funded by Fondazione CON IL SUD (Grant No. 2018-PDR-01004).
[1] S. H. Lee, X. Zhu, and W. D. Lu, Nanoscale resistive switching devices for memory and computing applications, Nano Res. 13, 1228 (2020).

[2] M. Di Ventra and F. L. Traversa, Perspective: Memcomputing: Leveraging memory and physics to compute efficiently, J. Appl. Phys. 123, 180901 (2018).

[3] D. Ielmini and H.-S. P. Wong, In-memory computing with resistive switching devices, Nat. Electron. 1, 333 (2018).
[4] F. L. Traversa and M. Di Ventra, Universal memcomputing machines, IEEE Trans. Neural Networks Learn. Syst. 26, 2702 (2015).

[5] A. V. Avizienis, H. O. Sillin, C. Martin-Olmos, H. H. Shieh, M. Aono, A. Z. Stieg, and J. K. Gimzewski, Neuromorphic atomic switch networks, PLoS One 7, e42772 (2012).

[6] R. A. Nawrocki, R. M. Voyles, and S. E. Shaheen, A mini review of neuromorphic architectures and implementations, IEEE Trans. Electron. Devices 63, 3819 (2016). 
[7] P. Borziak, V. Dyukov, A. Kostenko, Y. Kulyupin, and S. Nepijko, Electrical conductivity in structurally inhomogeneous discontinuous metal films, Thin Solid Films 36, 21 (1976).

[8] A. Sattar, S. Fostner, and S. A. Brown, Quantized Conductance and Switching in Percolating Nanoparticle Films, Phys. Rev. Lett. 111, 136808 (2013).

[9] C. Minnai, A. Bellacicca, S. A. Brown, and P. Milani, Facile fabrication of complex networks of memristive devices, Sci. Rep. 7, 7955 (2017).

[10] M. Mirigliano, F. Borghi, A. Podestà, A. Antidormi, L. Colombo, and P. Milani, Non-ohmic behavior and resistive switching of Au cluster-assembled films beyond the percolation threshold, Nanoscale Adv. 1, 3119 (2019).

[11] M. Mirigliano, D. Decastri, A. Pullia, D. Dellasega, A. Casu, A. Falqui, and P. Milani, Complex electrical spiking activity in resistive switching nanostructured Au two-terminal devices, Nanotechnology 31, 234001 (2020).

[12] M. Mirigliano, S. Radice, A. Falqui, A. Casu, F. Cavaliere, and P. Milani, Anomalous electrical conduction and negative temperature coefficient of resistance in nanostructured gold resistive switching films, Sci. Rep. 10, 19613 (2020).

[13] M. Mirigliano and P. Milani, Electrical conduction in nanogranular cluster-assembled metallic films, Adv. Phys. X 6, 1908847 (2021).

[14] W. Tarantino and L. Colombo, Modeling resistive switching in nanogranular metal films, Phys. Rev. Research 2, 043389 (2020).

[15] G. Benetti, C. Caddeo, C. Melis, G. Ferrini, C. Giannetti, N. Winckelmans, S. Bals, M. J. Van Bael, E. Cavaliere, L. Gavioli, and F. Banfi, Bottom-up mechanical nanometrology of granular Ag nanoparticles thin films, J. Phys. Chem. C 121, 22434 (2017).

[16] D. Gall, Electron mean free path in elemental metals, J. Appl. Phys. 119, 085101 (2016).

[17] F. Tavazza, D. T. Smith, L. E. Levine, J. R. Pratt, and A. M. Chaka, Electron Transport in Gold Nanowires: Stable 1-, 2- and 3-Dimensional Atomic Structures and Noninteger Conduction States, Phys. Rev. Lett. 107, 126802 (2011).

[18] S. Plimpton, Fast parallel algorithms for short-range molecular dynamics, J. Comput. Phys. 117, 1 (1995).

[19] H. Heinz, R. Vaia, B. Farmer, and R. Naik, Accurate simulation of surfaces and interfaces of face-centered cubic metals using 12-6 and 9-6 Lennard-Jones potentials, J. Phys. Chem. C 112, 17281 (2008).

[20] A. Stukowski, Visualization and analysis of atomistic simulation data with OVITO - the open visualization tool, Modell. Simul. Mater. Sci. Eng. 18, 015012 (2009).

[21] I. S. Beloborodov, A. V. Lopatin, V. M. Vinokur, and K. B. Efetov, Granular electronic systems, Rev. Mod. Phys. 79, 469 (2007).

[22] See Supplemental Material at http://link.aps.org/supplemental/ 10.1103/PhysRevMaterials.5.126001 for a detailed discussion on the importance of the different transport mechanisms, which includes Refs. [43-46].

[23] T. Kawamura and J. P. Leburton, Quantum ballistic transport through a double-bend waveguide structure: Effects of disorder, Phys. Rev. B 48, 8857 (1993).
[24] M. Dreher, F. Pauly, J. Heurich, J. C. Cuevas, E. Scheer, and P. Nielaba, Structure and conductance histogram of atomic-sized Au contacts, Phys. Rev. B 72, 075435 (2005).

[25] Y. Kurui, Y. Oshima, M. Okamoto, and K. Takayanagi, Conductance quantization and dequantization in gold nanowires due to multiple reflection at the interface, Phys. Rev. B 79, 165414 (2009).

[26] I. K. Yanson, O. I. Shklyarevskii, S. Csonka, H. van Kempen, S. Speller, A. I. Yanson, and J. M. van Ruitenbeek, Atomic-Size Oscillations in Conductance Histograms for Gold Nanowires and the Influence of Work Hardening, Phys. Rev. Lett. 95, 256806 (2005).

[27] Y. Oshima, K. Mouri, H. Hirayama, and K. Takayanagi, Development of a miniature STM holder for study of electronic conductance of metal nanowires in UHV-TEM, Surf. Sci. 531, 209 (2003)

[28] V. Rodrigues, T. Fuhrer, and D. Ugarte, Signature of Atomic Structure in the Quantum Conductance of Gold Nanowires, Phys. Rev. Lett. 85, 4124 (2000).

[29] M. Kiguchi, T. Konishi, and K. Murakoshi, Conductance bistability of gold nanowires at room temperature, Phys. Rev. B 73, 125406 (2006)

[30] R. Suzuki, M. Tsutsui, D. Miura, S. Kurokawa, and A. Sakai, Distribution of $1 G_{0}$ plateau length of Au contacts at room temperature, Jpn. J. Appl. Phys. 46, 3694 (2007).

[31] T. Kizuka, Atomic configuration and mechanical and electrical properties of stable gold wires of single-atom width, Phys. Rev. B 77, 155401 (2008).

[32] H. Yasuda and A. Sakai, Conductance of atomic-scale gold contacts under high-bias voltages, Phys. Rev. B 56, 1069 (1997).

[33] J. M. Soler, E. Artacho, J. D. Gale, A. García, J. Junquera, P. Ordejón, and D. Sánchez-Portal, The SIESTA method for $a b$ initio order- $N$ materials simulation, J. Phys.: Condens. Matter 14, 2745 (2002).

[34] M. Brandbyge, J.-L. Mozos, P. Ordejón, J. Taylor, and K. Stokbro, Density-functional method for nonequilibrium electron transport, Phys. Rev. B 65, 165401 (2002).

[35] R. Landauer, Electrical conductivity in inhomogeneous media, AIP Conf. Proc. 40, 2 (1978).

[36] D. Erts, H. Olin, L. Ryen, E. Olsson, and A. Thölén, Maxwell and Sharvin conductance in gold point contacts investigated using TEM-STM, Phys. Rev. B 61, 12725 (2000).

[37] D. Stauffer and A. Aharony, Introduction to Percolation Theory (Taylor \& Francis, Philadelphia, 1994).

[38] A. Maaroof and B. Evans, Onset of electrical conduction in $\mathrm{Pt}$ and Ni films, J. Appl. Phys. 76, 1047 (1994).

[39] F. Burgmann, S. Lim, D. McCulloch, B. Gan, K. Davies, D. R. McKenzie, and M. Bilek, Electrical conductivity as a measure of the continuity of titanium and vanadium thin films, Thin Solid Films 474, 341 (2005).

[40] E. Barborini, G. Corbelli, G. Bertolini, P. Repetto, M. Leccardi, S. Vinati, and P. Milani, The influence of nanoscale morphology on the resistivity of cluster-assembled nanostructured metallic thin films, New J. Phys. 12, 073001 (2010).

[41] Y. Namba, Resistivity and temperature coefficient of thin metal films with rough surface, Jpn. J. Appl. Phys. 9, 1326 (1970). 
[42] R. C. Munoz and C. Arenas, Size effects and charge transport in metals: Quantum theory of the resistivity of nanometric metallic structures arising from electron scattering by grain boundaries and by rough surfaces, Appl. Phys. Rev. 4, 011102 (2017).

[43] G. D. Barmparis, G. Kopidakis, and I. N. Remediakis, Shapedependent single-electron levels for Au nanoparticles, Materials 9, 301 (2016).
[44] S. Mukhopadhyay and J. Peixinho, Packings of deformable spheres, Phys. Rev. E 84, 011302 (2011).

[45] I. S. Beloborodov, K. B. Efetov, A. V. Lopatin, and V. M. Vinokur, Transport Properties of Granular Metals at Low Temperatures, Phys. Rev. Lett. 91, 246801 (2003).

[46] I. S. Beloborodov, A. V. Lopatin, and V. M. Vinokur, Universal description of granular metals at low temperatures: Granular Fermi liquid, Phys. Rev. B 70, 205120 (2004). 